\title{
DESCRIPTION OF HYDRAULIC AND STRENGTH PROPERTIES OF ANISOTROPIC GEOMATERIALS
}

\author{
S. PIETRUSZCZAK \\ McMaster University, Hamilton, Ont. Canada L8S 4L7, \\ e-mail: pietrusz@mcmaster.ca \\ G.N. PANDE \\ Swansea University, Swansea, U.K., \\ e-mail: g.n.pande@swansea.ac.uk
}

\begin{abstract}
In this paper, a simple generalization of Darcy's law is proposed for the description of hydraulic properties of anisotropic porous materials. The coefficient of permeability is defined as a scalarvalued function of orientation. The principal directions of permeability are determined from a fabric descriptor specifying the distribution of average pore size. An example is provided for identification of material parameters, which is based on an idealized "pipe network model". A procedure for defining the anisotropy in strength properties, which incorporates a conceptually similar approach, is also reviewed and an illustrative example is provided.
\end{abstract}

\section{INTRODUCTION}

Many naturally occurring geomaterials are anisotropic at the macroscale. The anisotropy manifests itself in the directional dependence of both hydraulic as well as mechanical properties. In granular materials, the anisotropy may stem from the shape of the grains. It is well known, for example, that the strength properties of sand with elongated angular-shaped aggregates are strongly affected by the direction of loading in relation to the direction of deposition (cf. Oda et al. [8]). But even if the skeleton itself may be considered as isotropic, e.g., sand with nearly spherical particles, there is usually a bias in the distribution of void space, due to initial compaction, which also triggers the anisotropy that may evolve during the deformation process. In sedimentary rocks (like shales) there is typically a strong inherent anisotropy, which is the result of the presence of bedding planes (cf. Niandou et al. [7]); the latter clearly visible at the macroscale. Whether inherent, induced or a combination of both, the anisotropy in microstructure may have a significant effect in the context of analysis and design of geotechnical structures.

This paper addresses the issue of mathematical description of anisotropy in hydraulic as well as strength properties of geomaterials. A common framework is adopted for both these cases, which incorporates some scalar-valued functions defined over a unit sphere and represented in terms of a traceless second-order tensor. The parameters appearing in this representation are identified either from a computational 
procedure or the experimental data. Section 2 deals with description of anisotropy of permeability. The material fabric is defined by incorporating the notion of "areal pore size". The definition of this descriptor is based on linear intercept measurements. A pipe network model, in which the porosity is represented by a cubic lattice of pipes of average pore diameters, is used to identify coefficients appearing in the function defining the permeability distribution. Section 3 deals with anisotropy of strength parameters. The methodology employed there incorporates the "critical plane" approach. The latter, once again, invokes a spatial distribution of strength parameters and the orientation of the critical plane is determined by maximizing the failure function with respect to the orientation. A numerical example is provided illustrating the procedure for identification of material parameters. Section 4 gives final remarks and offers some brief comments on future research.

\section{HYDRAULIC PROPERTIES; DARCY'S LAW FOR ANISOTROPIC POROUS MATERIALS}

It is postulated that for porous materials, which display anisotropy in terms of void space distribution, Darcy's law may be generalized to

$$
\mathrm{v}_{i}=k\left(n_{i}\right) i_{i} ; \quad i_{i}=\frac{\partial h}{\partial x_{i}} ; \quad n_{i}=\frac{\mathrm{v}_{i}}{\left(\mathrm{v}_{k} \mathrm{v}_{k}\right)^{1 / 2}}
$$

where $\mathrm{v}_{i}$ is the apparent velocity of fluid, $h$ is the total hydraulic head and the coefficient of permeability (often referred to as hydraulic conductivity) $k$ is taken as a function of the orientation of the sample relative to the direction of flow, i.e.,

$$
k\left(n_{i}\right)=k_{0}\left[1+\Omega_{i j} n_{i} n_{j}+a_{1}\left(\Omega_{i j} n_{i} n_{j}\right)^{2}+a_{2}\left(\Omega_{i j} n_{i} n_{j}\right)^{3}+\ldots\right] .
$$

Here, the coefficients $a_{1}, a_{2}, \ldots$ as well as the parameter $k_{0}$ are constants, while $\Omega_{i j}$ is a symmetric traceless tensor that describes the bias in the directional distribution of permeability.

In order to define the principal directions of permeability a measure of material microstructure is required. Since the hydraulic properties depend on both the size and the distribution of void space, the specific descriptor adopted here is the "areal pore size" $\rho_{\mathrm{A}}$, which is analogous to that introduced in earlier work by Pietruszczak and Krucinski [10] and Inglis and Pietruszczak [5]. The definition is based on principles of stereology and employs linear intercept measurements. Consider first an arbitrary plane with unit normal $v_{i}$ passing through REV that is perceived as a sphere with a radius $R$. Introduce now a set of uniformly distributed parallel lines aligned in an arbitrary direction $\omega_{i}$ (i.e. $\left.v_{i} \omega_{i}=0\right)$ that covers a range of orientations $C \subset(0,2 \pi)$. The areal pore size on this plane is defined as 


$$
\bar{\rho}_{A}=\frac{1}{2 \pi} \int_{C\left(v_{i}\right)} g\left(\omega_{i}\right) d C ; \quad g\left(\omega_{i}\right)=\frac{\sum I\left(\omega_{i}\right)}{N\left(\omega_{i}\right)} .
$$

Here, $\sum I\left(\omega_{i}\right)$ is the sum of intercept lengths of all segments contained within the pore space, $N\left(\omega_{i}\right)$ is the number of intercepts, $d C$ is the differential angle measured in this plane and $g\left(\omega_{i}\right)=-g\left(\omega_{i}\right)$ is taken as symmetric with respect to the origin.

Given equation (3), the "areal pore size" descriptor $\rho_{\mathrm{A}}$ associated with the direction $v_{i}$ is now defined by averaging $\bar{\rho}_{\mathrm{A}}$ along $v_{i}$, i.e.,

$$
\rho_{A}\left(v_{i}\right)=\frac{1}{2 R} \int_{-R}^{R} \bar{\rho}_{A}\left(v_{i}\right) d L
$$

where $d L$ is the differential distance measured along $v_{i}$. Note that the integration in equation (4) can be replaced by a discrete summation. For this purpose, consider a set of equally spaced parallel test lines on any set of $m$ equally spaced parallel planes. The test lines are rotated in-plane $k$ times around a pole defined by $v_{i}$. Then $\rho_{\mathrm{A}}$ can be defined in terms of discrete quantities $I$ and $N$, as

$$
\rho_{\mathrm{A}}\left(v_{i}\right)=\frac{1}{m} \sum_{m} \rho_{A}^{(m)}\left(v_{i}\right) ; \quad \rho_{\mathrm{A}}^{(m)}\left(v_{i}\right)=\frac{1}{k} \sum_{k} \frac{\sum I\left(\omega_{i}^{(k)}\right)}{N\left(\omega_{i}^{(k)}\right)} .
$$

The distribution (5) can be approximated using a representation similar to that of equation (2), i.e.,

$$
\rho_{A}\left(v_{i}\right)=c_{0}\left[1+D_{i j} v_{i} v_{j}+c_{1}\left(D_{i j} v_{i} v_{j}\right)^{2}+c_{2}\left(D_{i j} v_{i} v_{j}\right)^{3}+\ldots\right] .
$$

Given now a discrete set of data generated for an REV, the components of $D_{i j}$ as well as the coefficients $c_{0}, c_{1}, c_{2}, \ldots$ can be identified from the best-fit approximation employing representation (6). The principal directions of fabric are then obtained by solving the eigenvalue problem

$$
\left(D_{i j}-\lambda \delta_{i j}\right) e_{j}=0
$$

so that

$$
D_{i j}=\lambda_{1} e_{i}^{(1)} e_{j}^{(1)}+\lambda_{2} e_{i}^{(2)} e_{j}^{(2)}+\lambda_{3} e_{i}^{(3)} e_{j}^{(3)}
$$

where $\lambda$ 's are the eigenvalues of $D_{i j}$. Note that for granular materials $e_{i}^{(k)}(k=1,2,3)$ are the same for both operators $\Omega_{i j}$ and $D_{i j}$. Furthermore, the eigenvalues of $\Omega_{i j}$ and $D_{i j}$ may be related by employing some micromechanical estimates, as for an isotropic material $k=k\left(\rho_{\mathrm{A}}\right)$. 


\subsection{NUMERICAL EXAMPLES}

The identification of the permeability function (2) requires first the specification of principal axes of anisotropy, viz. equation (7). It is noted that these are not necessarily coaxial with those for anisotropy of strength, though there is likely to be a strong correlation between the two. Once the principal directions of permeability have been defined, the spatial distribution can be determined experimentally by performing tests on samples with different orientation with respect to the direction of flow. For granular materials, for example, this would entail a series of constant/falling head permeability tests. In the absence of the experimental data, the distribution of permeability may be assessed by means of some approximate numerical techniques. In order to provide an illustration, a simple "pipe network" representation of pore-structure, as used in other fields of engineering (cf. Chatzis and Dullien [2]; Diaz et al. [3], Kralj and Pande [6]), is employed here. Within the pipe network model, a single or multiphase flow is visualised as taking place through a network of inter-connected miniature pipes. Obviously, in practical terms, it is hardly possible to capture pore architecture and tortuosity in detail and drastic simplifications are required. Here, the pipes representing the pore space are arranged in a cubic lattice in such a way that their volume fraction corresponds to the porosity of the material and variations in diameter correspond to the pore size distribution. Figure 1 shows a typical network formed by a cubic lattice having a grid of $12 \times 12 \times 12$ with six pipes, of the same length, connecting at each node. It is obvious that as the grid size is increased a more accurate representation of flow and permeability will be achieved.

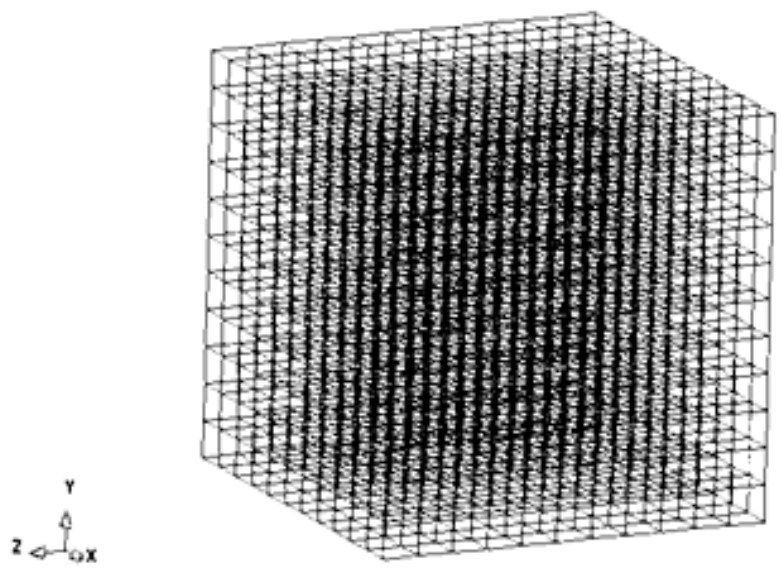

Fig. 1. Pipe network model representation

The variation in diameters of pipes in the network is represented by a log-normal distribution having a certain mean diameter $\bar{d}$ and an assigned standard deviation $\sigma$. 
Pipe diameters are allocated randomly. A fluid pressure is applied on a face of the cube and the infiltration of the fluid to the opposite face is traced layer by layer until a steady state flow is established through the sample. The lateral sides of the cube are assumed to be impermeable so that fluid flow out of the network is only achieved through the opposite face. Depending on the diameter and the characteristics of the permeating fluid, a specific pipe may or may not be penetrated. Percolation through the network is carried out in a series of iterations; in each cycle a pipe adjacent to a node with a pipe filled with fluid, may or may not allow fluid to pass depending on the pipe diameter. The smallest diameter necessary for percolation is calculated from the Washburn [12] equation

$$
p_{c}=p_{f}-p_{A}=\frac{4 T \cos \theta}{d}
$$

where $p_{c}$ is the capillary pressure, $p_{f}$ is the pressure at the node filled with fluid (water), $p_{A}$ is the pressure at an empty node (air), $T$ is the surface tension, $\theta$ is the contact angle and $d$ is the pipe diameter. Note that the latter may be identified here with the average "areal pore size" as defined viz. equation (6), i.e., $d \equiv \rho_{\mathrm{A}}$. If the fluid is unable to penetrate the pipe, then a "dead-end" condition is reached along that particular flow path. The flow is considered to be purely laminar and therefore the Hagen-Poiseuille equation (cf. Sutera and Skalak [11]) can be used for the calculations of pressure, i.e.,

$$
\Delta H=\frac{128 \mu l Q}{\gamma \pi d^{4}}
$$

where $\Delta H$ is the head loss along the pipe, $\mu$ is the viscosity of the fluid, $l$ is the length of the pipe, $Q$ is the flow rate through the pipe and $\gamma$ is the unit weight of fluid.

Once equilibrium has been reached and a constant flow through the sample has been established, then Darcy's law is used to compute the coefficient of permeability. As an example, Fig. 2 shows the variation of permeability with weighted average pore diameter along the direction of flow. Here, the coefficient of variation $\left(c_{v}=\sigma / \bar{d}\right)$ of pore diameters has been kept constant at 1.45 , while the coefficient of permeability has been normalized with respect to the value computed for the weighted mean pore diameter of $27.5 \mu \mathrm{m}$, i.e., $k \simeq 4 * 10^{-6} \mathrm{~m} / \mathrm{s}$.

Another study was carried out to assess the sensitivity of permeability to a more variable pore size distribution whilst keeping mean pore diameter as constant at $25.15 \mu \mathrm{m}$. Figure 3 shows the change in permeability with the coefficient of variation, the latter within the range between zero and 2.0. Permeability has been normalized here with respect to the value corresponding to coefficient of variation equal to zero. It is evident that increasing the coefficient of variation from zero to 2.0 leads to an $80 \%$ reduction in permeability demonstrating that this is a very sensitive parameter. 


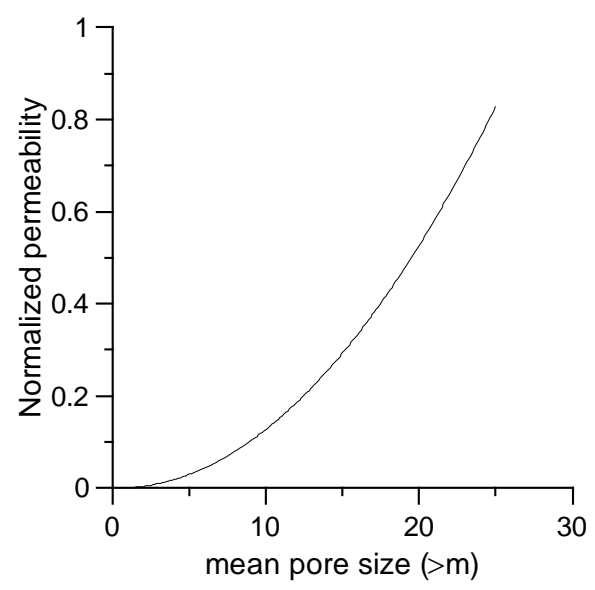

Fig. 2. Variation of permeability (normalized with respect to that corresponding to $d=27.5 \mu \mathrm{m}$ ) with the average pore size $\left(c_{v}=1.45\right)$

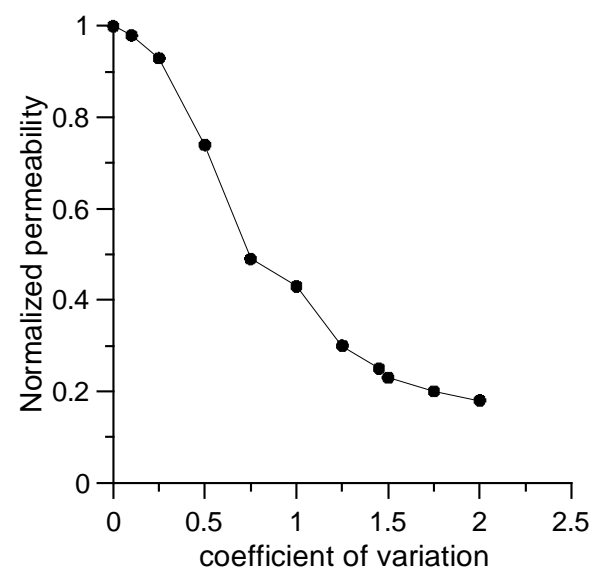

Fig. 3. Change in permeability with increasing coefficient of variation

Consider now a sample whose "areal pore size" distribution is defined by a linear form of representation (6), i.e., $c_{1}=c_{2}=\ldots=0$. Referring the problem to the principal material axes $\left\{x_{1}, x_{2}, x_{3}\right\}$, as shown in Fig. 4, assume that the microstructure displays a transverse isotropy, so that $D_{1}=D_{3}$. In this case, since $D_{i j}$ is traceless, there is

$$
\rho_{\mathrm{A}}\left(v_{i}\right)=c_{0}\left(1+D_{i j} v_{i} v_{j}\right)=c_{0}\left(1+D_{i j}\left(1-3 \cos ^{2} \alpha\right)\right) .
$$

Let the "areal pore size" in the vertical direction $(\alpha=0)$ be $\rho_{\mathrm{A}}(\alpha=0)=\rho_{\perp}=$ $15 \mu \mathrm{m}$, while that in the horizontal direction be $\rho_{\mathrm{A}}\left(\alpha=90^{\circ}\right)=\rho_{\perp}=27.5 \mu \mathrm{m}$. Substituting these values in equation (11) gives 


$$
c_{0}=\left(\rho_{O O}+2 \rho_{\perp}\right) / 3 ; \quad D_{1}=\rho_{\perp} / c_{0}-1
$$

so that $c_{0}=23.33 \mu \mathrm{m}, D_{1}=0.1786$. The second plot in Fig. 4 shows the distribution of "areal pore size" normalized with respect to the value at $\alpha=90^{\circ}$.

Given the information on the directional pore size distribution, the corresponding variation in permeability may now be established. For a vertical steady-state flow through an inclined sample, given the "areal pore size" in the direction of flow, the coefficient of permeability may be estimated directly from the distribution in Fig. 2. The results, for different orientations $\alpha$, are shown as black squares in Fig. 4. The same plot gives the best-fit approximations based on representation (2). The broken line corresponds to linear form of equation (2), i.e., $a_{1}=a_{2}=\ldots=0$, while the solid line represents the approximation in which the second order term has been retained. The latter gives the coefficients of approximation as

$$
k_{0}=0.697, \quad a_{1}=0.203, \quad \Omega_{1}=0.203 .
$$

Finally, Fig. 5 shows a rose diagram of normalized permeability associated with "areal pore size diameter" varying from 27.5 microns in the horizontal direction to 15 microns in the vertical direction. It is clear from this that, in a boundary value problem, the porosity of the material as well as the average pore size and its directional variation will both play a crucial role in determining the hydraulic characteristics of a porous medium.
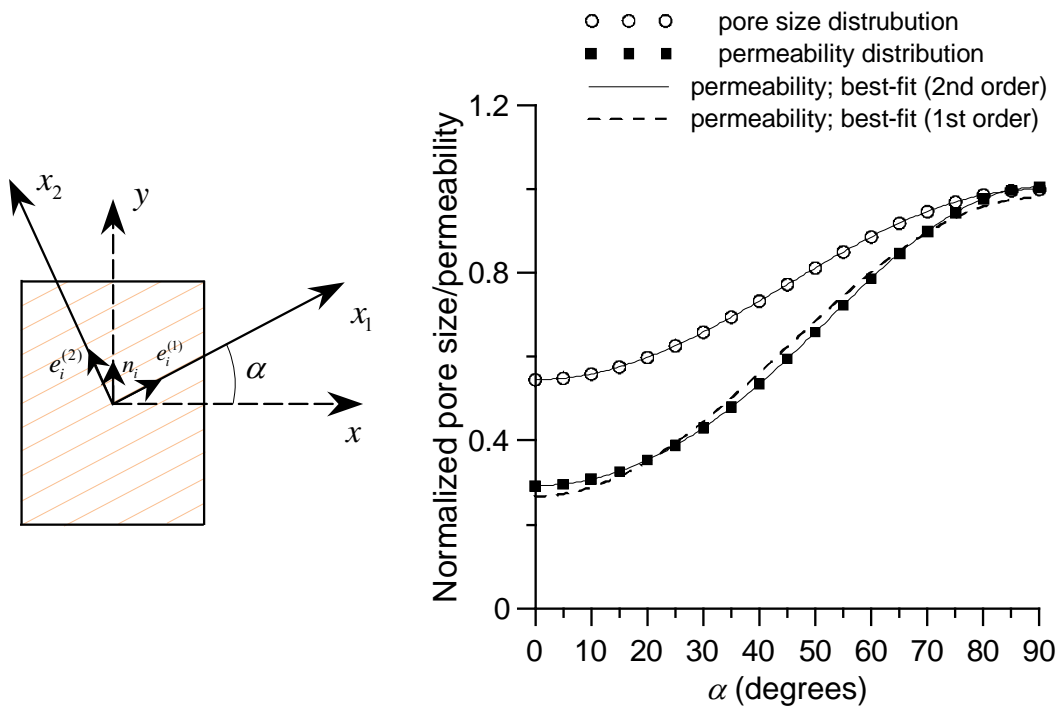

Fig. 4. Spatial variation of "areal pore size" and coefficient of permeability, normalized with respect to the values corresponding to $\rho_{\mathrm{A}}=27.5 \mu \mathrm{m}$ 


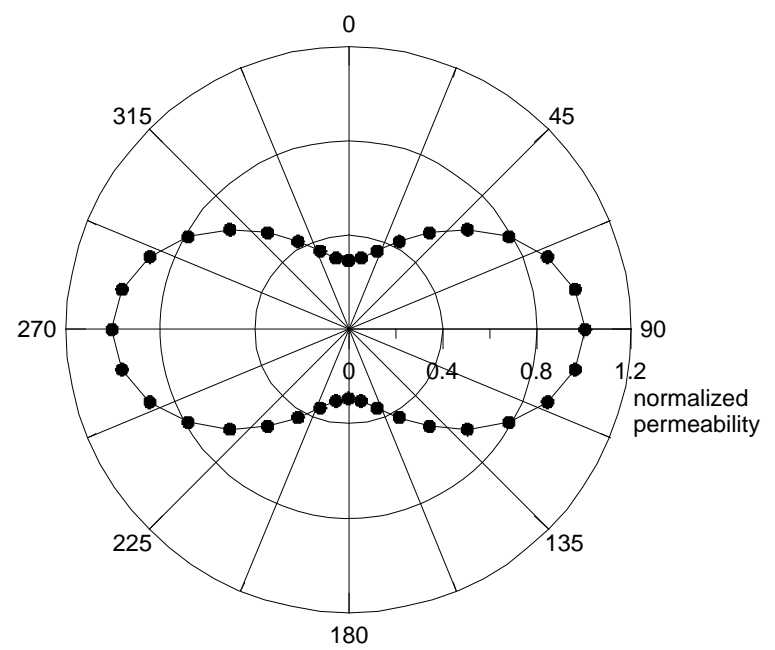

Fig. 5. Polar plot showing the spatial distribution of normalized permeability

\section{STRENGTH PROPERTIES; CRITICAL PLANE APPROACH}

The primary factor governing the mechanical response of inherently anisotropic media is the directional-dependence of strength properties. A proper description requires a specification of conditions at failure for an arbitrary orientation of microstructure under any given stress state. Over the last few decades, numerous failure criteria have been proposed and a review of different methodologies can be found, for example, in Duveau et al. [4] and/or Pietruszczak [9]. The methodology reviewed here incorporates the so-called "critical plane" approach. Within this framework, the failure criterion is defined in terms of traction components acting on the critical/localization plane. The approach employs a spatial distribution of strength parameters and the orientation of the critical plane is determined by maximizing the failure function with respect to the orientation. In order to illustrate the methodology, assume that the conditions at failure are governed by Coulomb criterion

$$
F=\tau-\mu \sigma-c=0 ; \quad \mu=\mu\left(n_{i}\right), \quad c=c\left(n_{i}\right) .
$$

In the equation above

$$
\sigma=\sigma_{i j} n_{i} n_{j}, \quad \tau=\left|\sigma_{i j} n_{j} s_{i}\right|, \quad n_{i} n_{i}=1, \quad n_{i} s_{i}=0
$$

where $\tau, \sigma$ represent the shear and normal components of the traction vector on a plane with unit normal $n_{i}$ and $\mu$ and $c$ are both said to be orientation dependent. The distribution of strength parameters $\mu=\tan \phi$ and $c$ can be described by employing a representation similar to that in equation (2), i.e., 


$$
\begin{aligned}
& \mu=\hat{\mu}\left(1+\Omega_{i j}^{\mu} n_{i} n_{j}+b_{1}\left(\Omega_{i j}^{\mu} n_{i} n_{j}\right)^{2}+b_{2}\left(\Omega_{i j}^{\mu} n_{i} n_{j}\right)^{3}+b_{3}\left(\Omega_{i j}^{\mu} n_{i} n_{j}\right)^{4}+\ldots\right), \\
& c=\hat{c}\left(1+\Omega_{i j}^{c} n_{i} n_{j}+d_{1}\left(\Omega_{i j}^{c} n_{i} n_{j}\right)^{2}+d_{2}\left(\Omega_{i j}^{c} n_{i} n_{j}\right)^{3}+d_{3}\left(\Omega_{i j}^{c} n_{i} n_{j}\right)^{4}+\ldots\right),
\end{aligned}
$$

where $\Omega$ 's are again symmetric second-order traceless tensors while the coefficients $b$ 's and $c$ 's, as well as the parameters $\hat{\mu}, \hat{c}$, are constants.

The problem of onset of failure and specification of the orientation of the critical plane can be formulated as a constrained optimization problem, i.e.,

$$
\max _{n_{i}, s_{i}} F=\max _{n_{i}, s_{i}}(\tau-\mu \sigma-c)=0 ; \quad n_{i} n_{j}=1, \quad n_{i} s_{i}=0, \quad s_{i} s_{i}=1 .
$$

The latter can be solved by Lagrange multipliers or any other suitable optimization technique. The solution provides the orientation of the critical/localization plane and defines the conditions at which the failure occurs under an arbitrary stress state.

For geomaterials with a strong inherent anisotropy (e.g., sedimentary rocks, sand with elongated angular-shaped particles, etc.), the principal material directions will not be significantly affected by the deformation process. On the other hand, if the initial anisotropy of fabric is weak (e.g., sand with nearly spherical particles), the preferred directions of the material, and thus the eigenvectors of $\Omega_{i j}$ 's will evolve in the course of the deformation process. In the latter case, one could argue that the microstructure is linked with the spatial distribution of voids. Since the rearrangement of void space is triggered by strain, one could postulate a simple evolution law that assumes a coaxiality of $\Omega_{i j}$ and $e_{i j}$, where $e_{i j}$ is the strain deviator. In this case, the spectral decomposition of $\Omega_{i j}$ takes the form

$$
\Omega_{i j}=\Omega_{1}^{0} m_{i}^{(1)} m_{j}^{(1)}+\Omega_{2}^{0} m_{i}^{(2)} m_{j}^{(2)}+\Omega_{1}^{0} m_{i}^{(3)} m_{j}^{(3)} ; \quad\left(e_{i j}-\lambda^{(k)}\right) m_{i}^{(k)}=0, \quad k=1,2,3
$$

where the eigenvalues are constant, while the principal directions $m_{i}^{(k)}(k=1,2,3)$ are obtained from the solution to an eigenvalue problem, as stated in equation (17). Obviously, this simple evolution law requires an appropriate verification. The latter would, in general, entail invoking the principles of stereology, via linear intercept measurements, similar to those discussed in the previous section. Note that the representation (17) implies that in standard material tests (such as those under biaxial/triaxial conditions), the principal directions of $\Omega_{i j}$ 's remain fixed, while under more complex strain trajectories, like those encountered in a boundary-value problem, they will progressively evolve.

\subsection{NUMERICAL EXAMPLES}

In order to illustrate the above framework consider the results of a series of direct shear tests performed on crushed limestone sand with elongated angular-shaped 
particles, as reported in the article by Azami et al. [1]. The samples were prepared by the sand rain method to void ratio of approx. 0.6, and were tested at different deposition angles $\alpha$ ranging from $0^{\circ}$ (horizontally aligned particles) to $90^{\circ}$ (vertically aligned). Typical results are presented in Figs. 6 and 7, which show the mechanical response at normal stress of $25 \mathrm{kPa}$ and $50 \mathrm{kPa}$. Clearly, the material exhibits a significant degree of anisotropy in terms of strength. Figure 8a shows the best-fit approximations of Coulomb failure envelopes, equation (13), for samples prepared at $\alpha=0^{\circ}$ and $\alpha=90^{\circ}$. It is interesting to note that the material develops a residual cohesion, and thus a residual resistance to tension, due to interlocking of particles. A complete set of experimental data, in terms of variation of $\mu$ and $c$ with the deposition angle $\alpha$, is given in Figs. 8b and 8c. The identification of the critical plane framework requires the specification of coefficients appearing in representation (15), which in turn involves establishing the best fit approximations to experimental data.
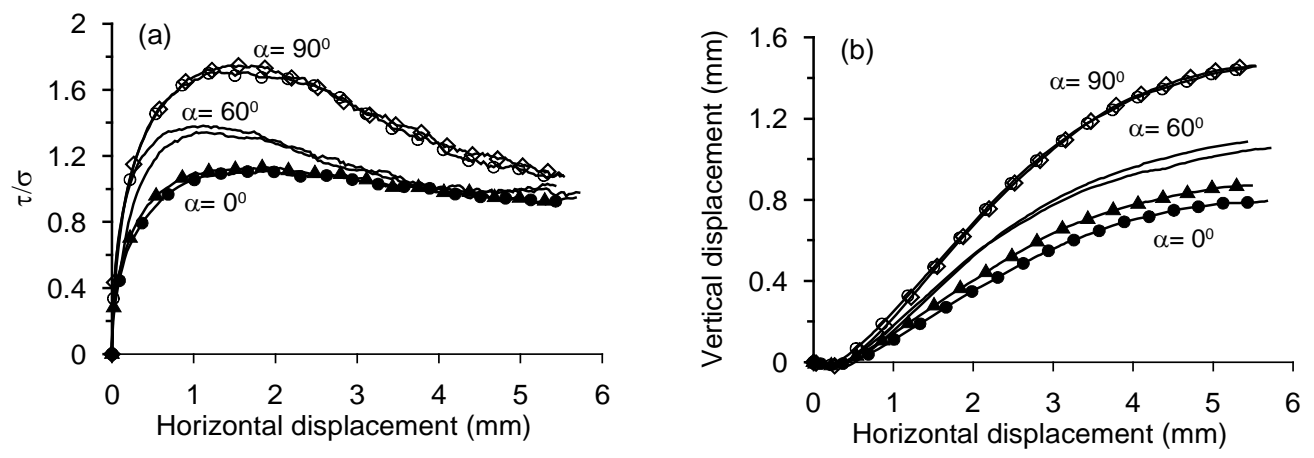

Fig. 6. Results of direct shear tests on crushed limestone sand (after ref. [1]) at normal stress of $25 \mathrm{kPa}$
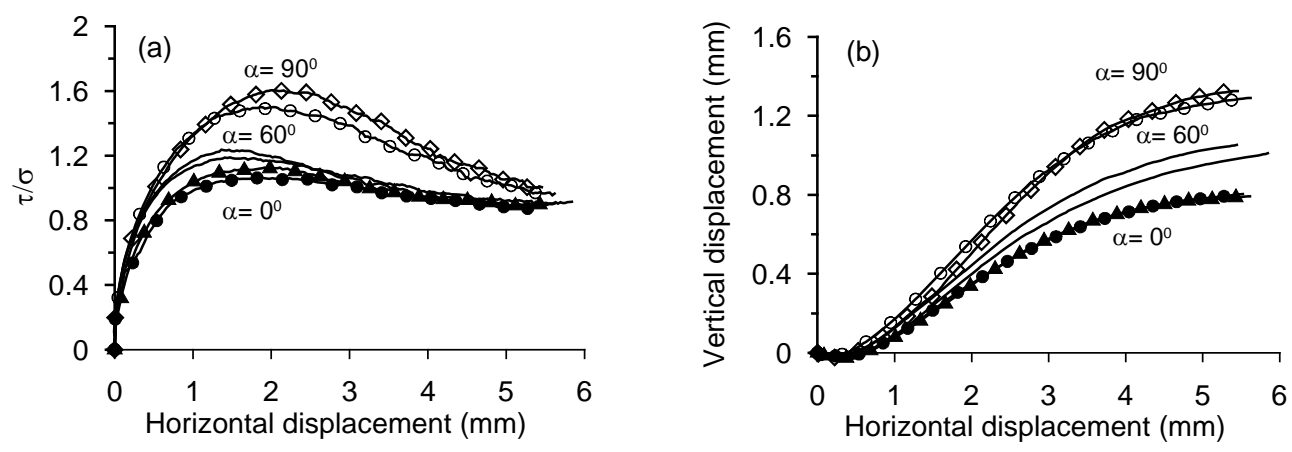

Fig. 7. Results of direct shear tests on crushed limestone sand (after ref. [1]) at normal stress of $50 \mathrm{kPa}$ 
(a)
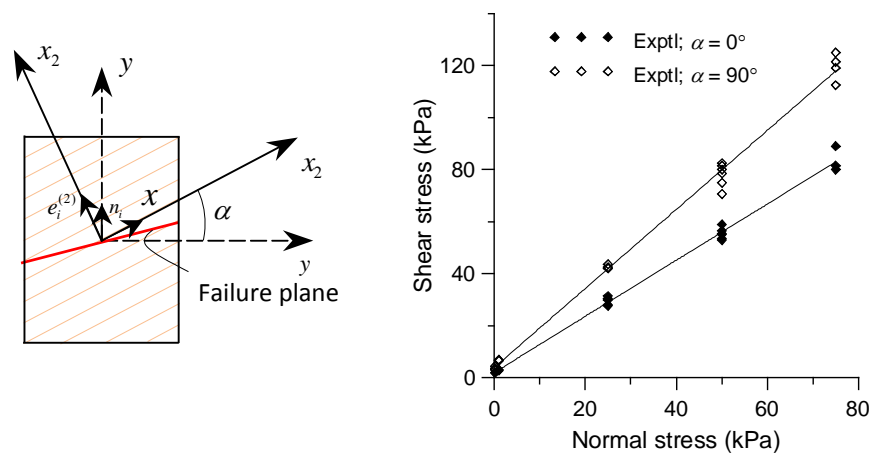

(b)

(c)
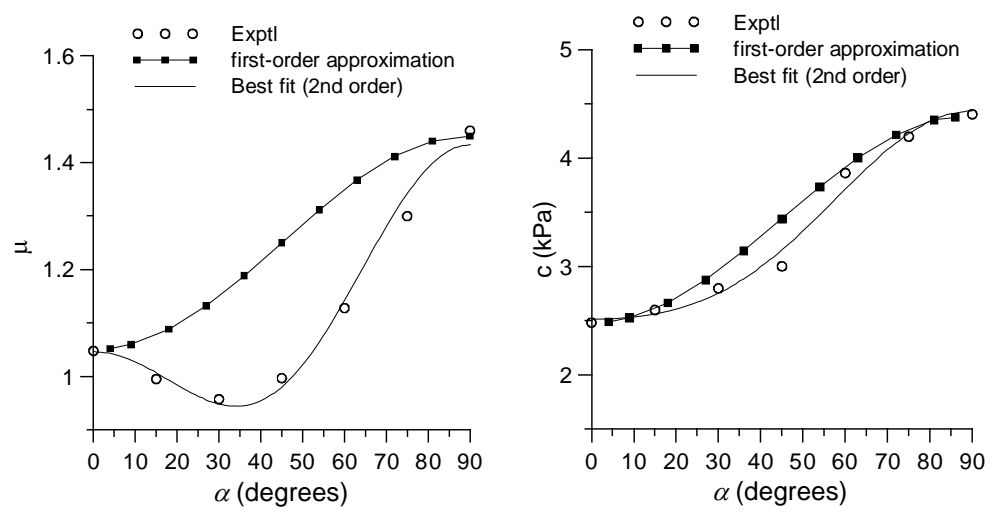

Fig. 8. (a) Best-fit approximations of Coulomb failure envelopes at $\alpha=0, \alpha=90^{\circ}$;

$(b, c)$ variation of friction coefficient $\mu$ and cohesion $c$ with the deposition angle

Apparently, if the higher order terms in the distribution of $\mu\left(n_{i}\right)$ and $c\left(n_{i}\right)$ are neglected, i.e., $b_{1}=b_{2}=\ldots=0$ and $d_{1}=d_{2}=\ldots=0$ in equations (15), the identification entails only the results for samples tested in the directions along and normal to the plane of material symmetry. Refer the problem to the coordinate system shown in Fig. 8. Here, by analogy to Fig. $4,\{x, y, z\}$ is the global frame of reference, while the base vectors $e_{i}^{(1)}, e_{i}^{(2)}, e_{i}^{(3)}$, associated with the coordinate axes $\left\{x_{1}, x_{2}, x_{3}\right\}$, define the principal material triad. In this case, $\Omega_{1}=\Omega_{3}$ and since $\Omega_{i j}$ is a traceless operator (i.e., $\Omega_{1}+\Omega_{2}+\Omega_{3}=0$ ), the following representation is obtained

$$
\mu\left(n_{i}\right)=\hat{\mu}\left(1+\Omega_{i j}^{\mu} n_{i} n_{j}\right)=\hat{\mu}\left(1+\Omega_{1}^{\mu} n_{1}^{2}+\Omega_{2}^{\mu} n_{2}^{2}+\Omega_{3}^{\mu} n_{3}^{2}\right)=\hat{\mu}\left(1+\Omega_{1}^{\mu}\left(1-3 \cos ^{2} \alpha\right)\right)
$$

with a similar representation holding for $c\left(n_{i}\right)$. Let the values of $\mu$ for samples tested at $\alpha=0^{\circ}$ and $\alpha=90^{\circ}$, be $\mu_{0}$ and $\mu_{\perp}$, respectively. Then, according to equation (18) 


$$
\hat{\mu}=\left(\mu_{0}+2 \mu_{\perp}=1.45 \Rightarrow \hat{\mu}=1.3, \Omega_{1}^{\mu}=0.1 .\right.
$$

Thus, given the experimental data in Fig. 8b, there is

$$
\mu_{0}=1.05, \quad \mu_{\perp}=1.45 \Rightarrow \hat{\mu}=1.3, \Omega_{1}^{\mu}=0.1 .
$$

The above approximation is not accurate, Fig. $8 \mathrm{~b}$, and better results are obtained by incorporating the higher order terms. Including, for example, the second degree term, i.e., approximating $\mu\left(n_{i}\right)$ as

$$
\mu\left(n_{i}\right)=\hat{\mu}\left(1+\Omega_{1}^{\mu}\left(1-3 \cos ^{2} \beta\right)+b_{1}\left(\Omega_{1}^{\mu}\left(1-3 \cos ^{2} \alpha\right)\right)^{2}\right)
$$

yields

$$
\hat{\mu}=1.07, \quad \Omega_{1}^{\mu}=0.23, \quad b_{1}=2.07
$$

and gives a much better representation. A similar conclusion is reached in the context of the bias in the spatial variation of cohesion, $c\left(n_{i}\right)$, Fig. $8 \mathrm{c}$. In this case, the secondorder approximation corresponds to

$$
c_{0}=3.51 \mathrm{kPa}, \quad \Omega_{1}=0.22, \quad b_{1}=0.82 .
$$

Given the coefficients of approximation (15), the framework defined viz. equation (16) can now be used in a predictive mode to define the conditions at failure under any possible combination of stress. Figure 9 shows the variation of axial compressive strength with the deposition angle $\beta$. The results of numerical simulations correspond to a series of "triaxial" tests conducted at the confinement of $10 \mathrm{kPa}$. The experimental data, which is taken again from ref. [1], gives the values corresponding to tests carried out at $\alpha=0^{\circ}$ and $\alpha=90^{\circ}$. It is quite evident that the effect of anisotropy is very significant as the compressive strength is reduced here from approx. $115 \mathrm{kPa}$ to $70 \mathrm{kPa}$.

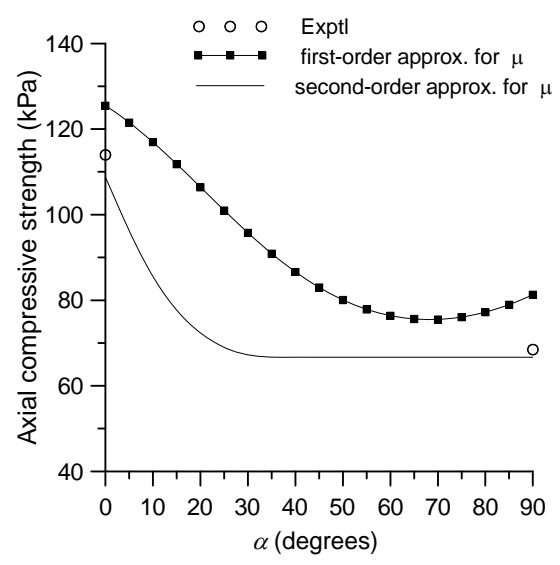

Fig. 9. Variation of axial compressive strength, at confining pressure of $10 \mathrm{kPa}$, with the deposition angle $\alpha$ 


\section{FINAL REMARKS}

A conceptually similar approach has been used here for describing the anisotropy in hydraulic and strength properties of porous media. It entails the specification of principal material directions. For certain geomaterials, e.g. sedimentary rocks, these are known a priori. On the other hand, for a more complex microstructural arrangement a specific fabric descriptor needs to be employed. For granular materials the inherent anisotropy is, in general, weak and may be altered in the course of deformation process. In this case, an appropriate relation is needed to define the evolution of the principal axes of microstructure. Specification of such an evolution law requires a continuous monitoring of the changes in structural arrangement, e.g., via computed tomography (CT) measurements, which is quite complex.

The scalar-valued functions describing the spatial variation of strength/hydraulic properties should ideally be identified from the experimental data. In the absence of such data, an implicit approach may be employed in which the relevant information is generated via a computational approach. Here, an illustrative example has been provided for specification of the distribution of permeability via a pipe network model. The pipe network model with cubic structure as used here may seem to be simplistic to represent the spatial variation of the pore space structure. However, it should be noted that three orthogonal directions chosen in the cubic network correspond to a point integration rule with an equal weight coefficient of $1 / 3$. This is the lowest order integration rule for a function varying over the surface of unit sphere. Higher order integration schemes will indeed lead to more complex pipe network and hopefully more accurate assessment of anisotropic permeability.

\section{REFERENCES}

[1] Azami A., Pietruszczak S., Guo P., Bearing capacity of shallow foundations in transversely isotropic granular media, Intern. Journ. Num. Anal. Meth. Geomech., 2009, 34(8), 771-793.

[2] Chatzis I., Dullien F.A.L., The modelling of mercury porosimetry and relative permeability of mercury in sandstones using percolation theory, Intern. Chem. Eng., 1985, 25, 47-66.

[3] Diaz C.E., Chatzis I., Dullien F.A.L., Simulation of capillary pressure curves using bond correlated site percolation on a simple cubic network, Transport in Porous Media, 1987, 2, 215-240.

[4] Duveau G., Shao J.F., Henry J.P., Assessment of some failure criteria for strongly anisotropic materials, Mech. Cohesive-Frictional Mater., 1998, 3, 1-26.

[5] Inglis D., Pietruszczak S., Characterization of anisotropy in porous media by means of linear intercept measurements, Intern. Journal of Solids \& Structures, 2003, 40, 1243-1264.

[6] Kralu B., Pande G.N., A stochastic model for the permeability characteristics of saturated cemented porous media undergoing freezing, Transport in Porous Media, 1996, 22, 345-357.

[7] Niandou H., Shao J.F., Henry J.P., Fourmaintraux D., Laboratory investigation of the mechanical behavior of Tournemire shale, Intern. Journ. Rock Mech. Min. Sci., 1997, 34, 3-16.

[8] Oda M., Koishikawa I., Huguchi T., Experimental study of anisotropic shear strength of sand by plane strain test, Soils and Foundations, 1978, 18(1), 25-38. 
[9] Pietruszczak S., Fundamentals of Plasticity in Geomechanics, Taylor \& Francis Group, Leiden/ London/New York, 2010.

[10] Pietruszczak S., KRUCiŃSKi S., Description of anisotropic response of clays using a tensorial measure of structural disorder, Mechanics of Materials, 1989, 8, 237-249.

[11] Sutera S.P., Skalak R., The history of Poiseuille's law, Annual Review of Fluid Mech., 1993, 25, $1-19$.

[12] WashbuRn E.W., The dynamics of capillary flow, Physical Review, 1921, 17(3), 273. 